IP Periodica Polytechnica Electrical Engineering and Computer Science

60(1), pp. 65-71, 2016

DOI: $10.3311 /$ PPee.9048

Creative Commons Attribution (i)

RESEARCH ARTICLE

\section{A Technique for Analyzing UWB Antennas Using the TLM Method}

\author{
Sebastian Held ${ }^{1}$, Markus Neinhüs ${ }^{2}$, Peter Waldow ${ }^{1}$, Adalbert Beyer ${ }^{3 *}$
}

Received 09 September 2015; accepted 22 January 2016

\begin{abstract}
In this paper, a technique for antenna analysis with the help of Transmission Line Matrix (TLM) Method is presented. In order to simulate a free space environment the TLM method requires the use of an absorbing boundary condition. The latter is realized by the EPML (extended perfectly matched layer). For the applicability of the technique proposed, a bow-tie and Vivaldi antenna are analyzed. Subsequently, numerical, as well as experimental results are introduced and discussed. These results obtained demonstrate the capability of this simulation technique for practical broad-band antenna design.
\end{abstract}

\section{Keywords}

Transmission Line Matrix (TLM) Method, extended perfectly matched layer (EPML), bow-tie antenna, Vivaldi antenna, reflection coefficient, radiation pattern

${ }^{1}$ IMST GmbH, 47475 Kamp-Lintfort, Carl-Friedrich-Gauß-Str. 2-4, Germany

${ }^{2}$ Rohde\&Schwarz, 51147 Köln, Graf-Zeppelin-Strasse 16, Germany

${ }^{3}$ Department of Electrical Engineering, Duisburg-Essen University, 47057 Duisburg, Bismarckstrasse 81, Germany

*Corresponding author, e-mail: adalbert.beyer@uni-due.de

\section{Introduction}

Until recently, the analysis and design of antennas in micro strip technology was often carried out by means of CAD (Computer Aided Design) or CAE (Computer Aided Engineering) programs. In these tools, the calculation method is based on analytical equations that are only valid up to a certain frequency. Normally, these analytical models describe a particular form of an antenna, only. The calculations take into account no surrounding elements, that is, the antenna is calculated as if it is floating freely in space. For the development of large scale integrated circuits, this assumption is a problem, because couplings between individual lines or structures are not modelled correctly. Especially, at high frequencies such errors occur.

Thus, for realistic description of an antenna, it is necessary to perform a simulation by using full-wave simulators. These techniques, depending on the technique chosen, can then calculate any complicated structures. For example, the Agilent Momentum has integrated in its circuit simulator ADS (Advanced Design System) the full-wave simulator, which is based on the method of moments. Just as the commercial simulator Sonnet, this can only simulate planar structures. Both simulators operate in the frequency domain and, therefore, they allow only the analysis of the steady state. Hence, transients cannot be investigated. To the class of the frequency domain method belongs also the FEM (Finite Element Method) method that is applied in the simulator HFSS (High Frequency Structure Simulator) of Ansoft. This simulator is to investigate complex three-dimensional objects in the situation. The general disadvantage of frequency-based methods lies in the complex determination of frequency dependent variables, because these methods can calculate the structures only for a certain frequency per simulation run.

For this reason, a time domain method to analyze the microstrip antenna is used in this work. The two dominant methods are here the FDTD- (Finite Difference Time Domain) and the TLM (Transmission Line Matrix) Method. FDTD calculates the Maxwell's equations directly by using a staggered lattice, in which the $\mathrm{E}$ and $\mathrm{H}$ field components are shifted both in space and in time. Therefore, it is possible to determine 
partial derivatives in discrete form, which are emerging in the Maxwell's equations. For example, this method is used in the simulator of IMST (Institute of Mobile and Satellite Technique) company. The TLM method is based on the Huygens' Principle, and it uses an orthogonal mesh combined by transmission lines for describing the propagation of elementary waves along these lines. For this reason, the TLM method is theoretically very simple, because with knowledge about the theory of transmission lines clear to understand. Another advantage of this technique compared to FDTD is that all field components are known at the same time. Both time domain methods enable to perform a transient analyse, and a simulation run covers the entire range of frequencies, as well. In addition, the structures can be arbitrarily complex. Thus, these techniques belong to the group of very suitable candidates for calculating any UWB antenna problem.

A disadvantage of the time-domain method compared to the methods in the frequency domain is that the simulation space must be limited by appropriate boundary operators. Especially, in the case of simulation of free space, certain difficulties can be raised.

In the following sections the necessary methods and tools for the simulation of antennas in UWB applications are explained using the TLM method. First, the mains features of the TLM method are described in Section 2, by means of which antennas for UWB purposes are characterized. Section 3 discusses the problem of modelling finite field areas. Hereto, the PML technique offers an advantageous solution. Section 4 shortly describes the practical views of antenna simulation. Subsequently, in Section 5 two UWB antenna types are analyzed by the methods discussed. Numerical and experimental results are presented and compared with literature data and measurements. A short conclusion summarizes the main points of this paper.

\section{Features of the TLM Method}

The TLM method is based on the Huygens' Principle. This indicates that every wave is formed from a superposition of elementary waves. Each point of the space is the starting point of these latter's. These new waves have the same frequency and phase velocity as the original ones. The Huygens' Principle is defined in the continuum that because of limited resources cannot be mapped directly in the computer. By following approximations, it is possible the Huygens' Principle in the computer to implement:

- discretization of space

- discretization of time

- limitation of the simulation space

The finite region of space, in which the simulation is to take place, will be filled with an orthogonal grid of transmission lines. The points, in which the transmission lines meet, called nodes or scattering centers. These form together with the directly connected lines the TLM-cells or nodes. The most common node is the Symmetrical Condensed Node ( $\mathrm{SCN})$. Figure 1 shows a representation of this node, as described by Johns (1984) [1].

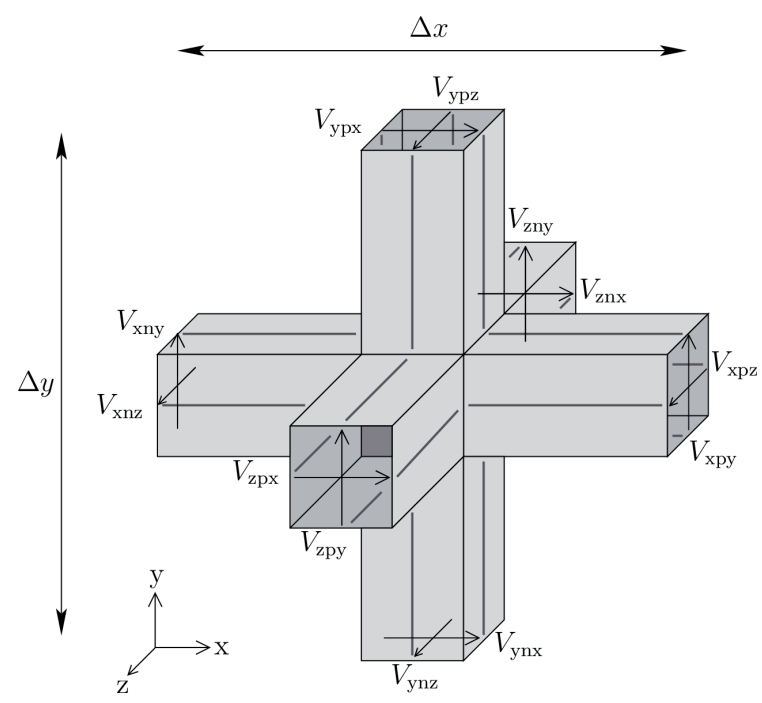

Fig. 1 SCN-TLM node introduced by Johns

It can be recognized that from each edge surfaces two orthogonal line pairs lead to the center of the node. If the ends of line pairs on the boundary surface are called as ports, and, at the same time, the voltages of these ports are defined, too, thus, the SCN can be referred to as 12-port junction. The voltages represent the tangential electric field components. The scattering matrix can be calculated by the condition of energy conservation and conservation of charge.

A node has the edge lengths of $\Delta \ell$ in each coordinate directions (equidistant or isotropic grid), or, $\Delta x, \Delta y$ and $\Delta \mathrm{z}$ in an anisotropic grid (s. Fig. 1). The parameter $\Delta \ell$ would correspond to a cubic-shaped cell, which is determined by the time step $\Delta \mathrm{t}$ (Courant criterion), and, it is given by

$$
\Delta t_{\max }=\frac{\Delta \ell_{\min }}{\sqrt{3 c_{0}}}=\frac{\Delta \ell_{\min }}{2 c_{0}}
$$

for $\Delta \ell=\Delta x=\Delta y=\Delta z$

with $\mathrm{c}_{0}$ the velocity of light in free space.

Up to now, several node types have been developed, which enable to the description of the wave propagation according to the Helmholtz equation [2]. In his above-mentioned publication, Johns used the so-called central description in order to determine the field components within a node. While this form has the advantage that all field components in the same point in space are calculated (that is not necessarily the geometric center of the node), but it is not an unambiguous assignment. For this reason, the edge surfaces are used. The SCN has six edge surfaces (s. Fig. 1). On each of these surfaces two E and two $\mathrm{H}$ components are calculated. 
In this paper, field components are excited or read exclusively on the edge surfaces. The fact that in the edge surfaces only four of the six field components can be calculated means no limitation.

\section{The Extended Perfectly Matched Layer (EPML)}

During the simulation of infinitely extended electromagnetic field areas several problems will occur due to finite resources of the computer. Following this, the computation area must be limited. This is especial the case in determining antenna properties. A finite simulation field always contains limitations on which reflections occur. The Extended Perfectly Matched Layer (EPML) now offers a way to avoid re flections in a way that the wave is damped. The principle of this technique is to absorb incident wave from any direction and at any frequency. This method is similar to a measurement within an anechoic chamber. The 3D FDTD PML and its implementation in TLM have been published by Bérenger [3], Dubard and Pompei [4], and Le Maguer and Ney [5].

\subsection{Theoretical Background}

As mentioned above, the PML should absorb free of reflection incident wave from any direction and at any frequency. Thus, it is necessary to introduce new degrees of freedom in the Maxwell equations. The first step is to form them to be symmetrical. This can be reached by introducing the magnetic current density $\vec{J}_{m}=\sigma_{m} \vec{H}$ into the Maxwell equation, with the quantity $\sigma_{m}$, which is the magnetic conductivity:

$$
\begin{aligned}
& \operatorname{rot} \vec{H}=\frac{\partial \vec{D}}{\partial t}+\vec{J}_{e} \\
& \operatorname{rot} \vec{E}=\frac{\partial \vec{B}}{\partial t}-\vec{J}_{m}
\end{aligned}
$$

It should be noted, however that both quantities introduced are nonphysical values.

The aim is to match the PML medium to the adjacent medium (vacuum or any material) that no reflections occur. For that purpose, incident wave travelling in a lossless material marked by (1) is subdivided into two parts. First, $\mathrm{TE}_{\mathrm{z}}$-portion of the wave $\left(\vec{E}^{i}, \vec{H}^{i}\right)$ is examined, which is penetrating into a lossy medium [6]. This case is also referred to as parallel, vertical or an H-polarization. $\mathrm{TE}_{\mathrm{z}}$ wave means that the vector $\vec{E}$ lies transversal to the $\mathrm{z}$ axis and the vector $\vec{H}$ possesses only one component in $\mathrm{z}$ direction. Figure 2 illustrates these facts.

For the phase constant following relationships apply in the area marked by (1):

$$
\beta_{1 x}=k_{1} \cos \Theta_{i} \text { and } \beta_{1 y}=k_{1} \sin \Theta_{i}
$$

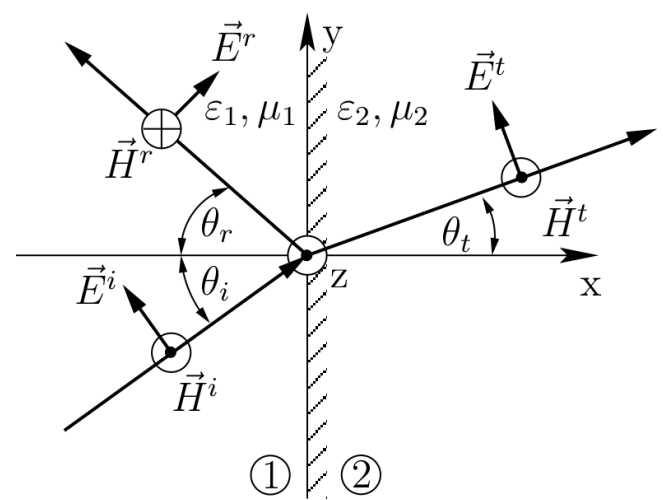

Fig. 2 Reflection and transmission of a TEz wave at a border between two different materials.

The relationship in the area marked by (2) is a bit more complicated, since electric and magnetic losses must be considered:

$$
\beta_{2 x}=\sqrt{k_{2}^{2}\left(1+\frac{\sigma_{e}}{j \omega \varepsilon_{2}}\right)\left(1+\frac{\sigma_{m}}{j \omega \mu_{2}}\right)-\beta_{2 y}^{2}}
$$

Here $k_{v}, v=1,2$ is the wave number, and, defined as

$$
k_{v}=\omega \sqrt{\mu_{v} \varepsilon_{v}} \text { for } v=1,2 .
$$

If the continuity of the tangential field components at the boundary layer $(x=0)$ is fulfilled, and only the normal incidence of the wave $\left(\theta_{i}=0\right)$ is considered, it follows for the reflection factor R:

$$
R=\frac{Z_{01}-Z_{02}}{Z_{01}+Z_{02}}
$$

For the characteristic wave impedances of the materials (1) and (2) the following expressions are valid:

$$
Z_{01}=\sqrt{\frac{\mu_{1}}{\varepsilon_{1}}} \text { and } Z_{02} \sqrt{\frac{\mu_{2}\left(1+\frac{\sigma_{m}}{j \omega \mu_{2}}\right)}{\varepsilon_{2}\left(1+\frac{\sigma_{e}}{j \omega \varepsilon_{2}}\right)}}
$$

Under certain circumstances, the reflection factor is vanishes (no reflections of the incident wave). For that case, the following conditions are necessary:

$$
\varepsilon_{1}=\varepsilon_{2}=\varepsilon, \quad \mu_{1}=\mu_{2}=\mu \quad \text { and } \quad \frac{\sigma_{e}}{\varepsilon}=\frac{\sigma_{m}}{\mu} .
$$

A medium with electric and magnetic losses marked by (2) In Fig. 2, of which material properties are in accordance with Eq. (9), is matched optimally to the material marked (1) for the case of normal incidence of a wave. The technique of Perfectly Matched Layer (PML) expands this optimum adaptation now to all angles of incidence for any types of waves. 


\subsection{Features of the PML technique}

To characterize a PML medium is the reflection factor $R(0)$ is used. It describes the expected reflection of a plane wave at normal incidence on a PML, which is terminated by an electric wall (Perfect Electric Conductor; PEC). According to [3], it yields:

$$
R(0)=\exp \left[-2 Z_{0} \int_{0}^{\delta} \sigma_{e}(\xi) d \xi\right] .
$$

These variables mean the following: $Z_{0}$ represents the characteristic wave impedance of the medium considered, and, $\delta$ stands for the thickness of PML. This value of $R(0)$ allows an estimation of the resultant reflection by the insufficient conductivity of PML. This value estimated is valid approximately, until numerical reflections take the upper hand, which occur by refining the discretization.

\subsection{Layered EPML}

As already mentioned, for simulation of an antenna discrete space is used, which may represent only an approximation of the continuous space. Therefore, the governing relationships are no longer differential equations, but difference equations. This fact has the consequence that abrupt changes in the conductivity of the media (e.g. vacuum-PML boundary layer) lead to unphysical reflections. For this reason it is not possible to reduce the PML to a width of only one cell, which might have a very high conductivity. Rather, one must construct a PML so that the jumps between the different conductivities remain low. Such a PML can be constructed by using several layers. It has been found by numerical experiments that a certain geometric distribution-function of the conductivities $\sigma_{e}$ and $\sigma_{m}$ delivers the best results. It yields:

$$
\sigma_{e, m}(\rho)=-\frac{\varepsilon_{0} c_{0}}{2 \Delta \ell} \frac{\ln g}{g^{N}-1} \ln R(0) g^{\rho / \Delta \ell} .
$$

The quantities here are: $\rho=$ distance from the vacuum-PML boundary layer, $\Delta \ell=$ resolution in direction towards vacuumPML boundary layer, $g=$ geometric order of the distribution $\left(g_{\text {opt }}=2.15\right), N=$ number of cells (thickness) of PML.

Below a certain threshold frequency undesirable reflections occur, even using the technique of Perfectly Matched Layer. This cut-off frequency is of numerical origin and, in the continuum undetectable. The reflections have their origin in the evanescent waves. These are absorbed more strongly than the propagating waves. Because of the discretization, it may happen that such an evanescent wave would be absorbed in a single cell, which is not possible by the algorithm, because the difference equations do not permit an abrupt absorption. The smaller the conductivity of first layer is, the lower the reflections are. However, the thickness of the entire PML must be selected to be greater, in order to achieve a specific reflection factor $R(0)$.

As already turned out, the PML must be built up containing several layers. The definition of a PML is by hand, even for a very small simulation space, virtually impossible. This can be recognized by the following example. Considering a very small volume of $10 \times 10 \times 10$ cells, which is enclosed by two absorbing layers ( $N=2)$ using $g_{\text {opt }}=2.15$ in Eq. (11), and with $R(0)=0.01$. In this example, altogether 804 TLM cells must be considered (due to the stepped structure). In each of these cells six conductivities $\left(\sigma_{e x}, \sigma_{e v}, \sigma_{e z}, \sigma_{m x}, \sigma_{m y}, \sigma_{m z}\right)$ have to be calculated. In order to overcome this difficulty, three different groups of cells can be defined.

1) For the group no. 1 (PML wall), the following statements are valid:

$$
\begin{array}{ll}
\sigma_{e z}=\sigma_{e z}(\rho), & \sigma_{m z}=\sigma_{e z} \frac{\mu}{\varepsilon}, \\
\sigma_{e x}=\sigma_{e y}=0, & \sigma_{m x}=\sigma_{m y}=0 .
\end{array}
$$

2) For the group no. 2 (PML edge) yields:

$$
\begin{aligned}
& \sigma_{e x}=\sigma_{e x}(\rho), \quad \sigma_{m x}=\sigma_{e x} \frac{\mu}{\varepsilon}, \quad \sigma_{e y}=\sigma_{y}(\rho), \\
& \sigma_{m y x}=\sigma_{e y} \frac{\mu}{\varepsilon}, \quad \sigma_{e z}=0, \quad \sigma_{m z}=0 .
\end{aligned}
$$

3) For the group no. 3 (PML corner) is obtained:

$$
\begin{array}{rlrl}
\sigma_{e x} & =\sigma_{e x}(\rho), & \sigma_{m x}=\sigma_{e x} \frac{\mu}{\varepsilon}, & \sigma_{e y}=\sigma_{e y}(\rho), \\
\sigma_{m y}=\sigma_{e y} \frac{\mu}{\varepsilon}, & \sigma_{e z}=\sigma_{e z}(\rho), & \sigma_{m z}=\sigma_{e z} \frac{\mu}{\varepsilon} .
\end{array}
$$

\subsection{EPML-SCN node}

During the numerical experiments, it turned out that by the help of a PML-SCN node suggested by Le Maguer and Ney in [5], the highest degree of stability can be reached for simulating a given antenna arrangement. It should be noted, however that into the governing equations two typing errors have crept in. After correcting them, the following equations are obtained for calculating the field sub-components for the time step $n \Delta t$ :

$$
\begin{gathered}
\Delta i E_{i j}^{(n)}=A_{e i j} C_{i j}\left(a_{j n i}+a_{j p i}+Y_{s i j} a_{e i j}-2 a_{e i k}\right), \\
\Delta i E_{i k}^{(n)}=A_{e i k} C_{i k}\left(a_{k n i}+a_{k p i}+Y_{s i k} a_{e i k}-2 a_{e i j}\right), \\
Z_{0} \Delta i H_{i j}^{(n)}=A_{m i j} D_{i j}\left(a_{j n k}-a_{j p k}+Z_{s i j} a_{m i j}-2 a_{m i k}\right), \\
Z_{0} \Delta i H_{i k}^{(n)}=A_{m i k} D_{i k}\left(-a_{k n j}+a_{k p j}+Z_{s i k} a_{m i k}-2 a_{m i j}\right) .
\end{gathered}
$$

The subscripts $i, j$ and $k$ stand for one of the following three cases:

$$
\begin{aligned}
& (i, j, k)=(x, y, z), \quad(i, j, k)=(\mathrm{y}, z, x), \\
& (i, j, k)=(z, x, y),
\end{aligned}
$$


By using the equivalences given by Eq. (19), twelve expressions for calculating all field components can be determined from the group of Eqs. (15)-(18). The other coefficients are defined as:

$$
\begin{gathered}
C_{i j}=\frac{c_{0} \Delta t \Delta i}{\varepsilon_{r i} \alpha_{j} \Delta j \Delta k}, \quad D_{i j}=\frac{c_{0} \Delta t \Delta i}{\mu_{r i} \alpha_{j} \Delta j \Delta k}, \\
Y_{s i j}=4\left(\frac{\varepsilon_{r i} \alpha_{j} \Delta j \Delta k}{2 c_{0} \Delta t \Delta i}-\frac{1}{2}\right), \quad Z_{s i j}=4\left(\frac{\mu_{r i} \alpha_{j} \Delta j \Delta k}{2 c_{0} \Delta t \Delta i}-\frac{1}{2}\right), \\
A_{e i j}=\frac{4}{4+G_{i j}}, \quad A_{m i j}=\frac{4}{4+R_{i j}}, \\
G_{i j}=Z_{0} \frac{2 \sigma_{e j} c_{0} \Delta t}{\varepsilon_{r i}}, \quad R_{i j}=Z_{0} \frac{2 \sigma_{m j} c_{0} \Delta t}{\mu_{r i}} .
\end{gathered}
$$

The value $\alpha$ describes the attenuation of the evanescent waves. The voltage pulses $a_{\text {inj }}$ and $a_{\text {ipj }}$ propagate to the center of the node in time step of $(\mathrm{n}-0.5) \Delta t$. Values $a_{\mathrm{eij}}$ and $a_{\mathrm{mij}}$ are the incident voltage pulses to the ports of the node. These 12 voltage pulses are added to the original 12 incident voltage pulses of the SCN node. All these voltage pulses have to be stored. Thus, the SCN-PML node needs twice the computer resources than the simple $\mathrm{SCN}$ node does.

\section{Antenna Simulations}

The validation of new antennas for UWB (ultra wide-band) communications can be accomplished using simulation techniques. Using the particular EPML implementation described above, the spatial distance between the AUT (antenna under test) and the surrounding absorber can be kept very small. In addition to that, attenuation of evanescent energy is considered. It has proven superior stability in antenna analysis.

All simulations were done using the SCN (Symmetrical Condensed Node) [1] with the condition: $\Delta \ell=\Delta x=\Delta y=\Delta z$. Also, a Near-to-Far-Field Transformation [7] was performed in order to get the antenna properties. The perfectly matched layer surrounds the computation domain. Its properties beside a geometric profile are:

Thickness $=5 \Delta \ell$, Reflection factor $R(0)=0.1 \%$, Evanescent damping $F=3$.

The latter is defined according to the expression introduced by [5] as:

$$
F_{z}=\frac{1}{\delta} \int_{0}^{\delta} \alpha_{z}(z) d z \quad \text { with } \quad \alpha_{z}(z)=1+\alpha_{\max }\left(\frac{z}{\delta}\right)^{n}
$$

The excitation is realized by a matched source feeding the micro strip structure. An SMA-connector generally used in measurements is omitted in the simulation. Extraction of scattering parameters is done with help of the algorithm of [8].

\subsection{Bow-Tie Antenna}

In order to demonstrate the effectiveness of this absorbing boundary condition, at first, the bow-tie antenna described by Kiminami [9] is restudied. The published results for the simulated frequency response differ from the measurement; therefore, a re-simulation was done. It should be mentioned, however that this type of antenna is often used for different millimetre-wave purposes, e.g. for monitoring sensors [10, 11].

The geometry of the analyzed double-sided printed bow-tie antenna is shown in Fig. 3a. This antenna was fabricated on Duroid substrate with a relative permittivity $\left(\varepsilon_{r}\right)$ of 6.15 and a thickness of $1.27 \mathrm{~mm}$. In contrast to the Simulation, it is equipped with a SMA-connector in order to fit easily with our HP8722C network analyzer. Thus, the reflection from the coaxmicro strip junction is not present in the simulation, but is kept as small as possible.

\subsection{Vivaldi Antenna}

This type of antenna was introduced and investigated in the 1980 's and 1990's, mainly for applying it for millimetre wave purposes. In this paper, an antipodal Vivaldi antenna $[12,13]$ (Fig. 3b) is examined. It is manufactured on Duroid $5870\left(\varepsilon_{r}=\right.$ 2.33, thickness $1.575 \mathrm{~mm}$ ). In contrast to [13], the antenna analyzed in this paper consists of only one substrate. Both types of the Vivaldi antenna are fabricated, but feeding the symmetric Vivaldi antenna (constructed out of two substrates glued together) was quite difficult. Hence, the physical properties of that antenna differ too much from the intended ones.

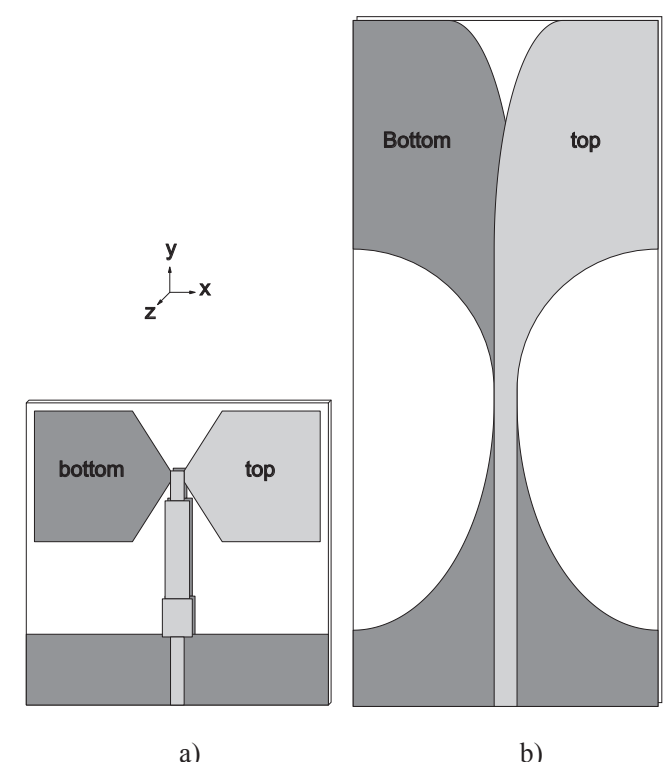

Fig. 3 Geometry of the bow-tie (a) and Vivaldi (b) antenna.

\section{Simulation Results}

The simulator is a self-written program based on TLM method using the EPML technique with the capability of calculating the reflection coefficient and the far-field radiation pattern in either frequency or time domain [6]. 


\subsection{Bow-tie antenna}

The magnitude of the reflection coefficient $\mathrm{S}_{11}$ is plotted in Fig. 4. It can be recognized that the values of simulated reflection coefficient reasonably agree with the measured ones. Compared to the results given by [9], the resonance at $7.4 \mathrm{GHz}$ is well predicted in the simulation. It is a common practice to consider an antenna matched, if the reflection coefficient is smaller than $-10 \mathrm{~dB}$. Hence, this antenna is usable between $4.5 \mathrm{GHz}$ $10 \mathrm{GHz}$, where the reflection coefficient is below $-10 \mathrm{~dB}$.

The azimuthal and elevation patterns are shown in Fig. 5 (a) and (b), respectively.

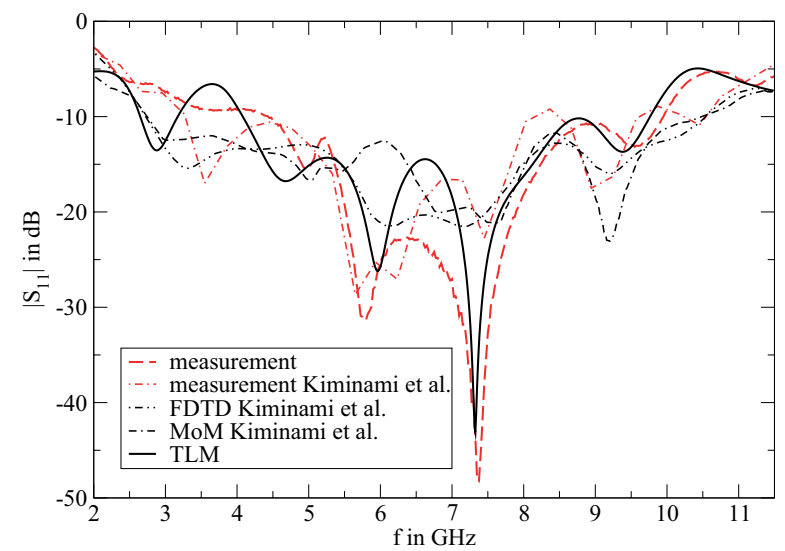

Fig. 4 Reflection coeflicient $\left|S_{11}\right|$ for the bow-tie antenna.

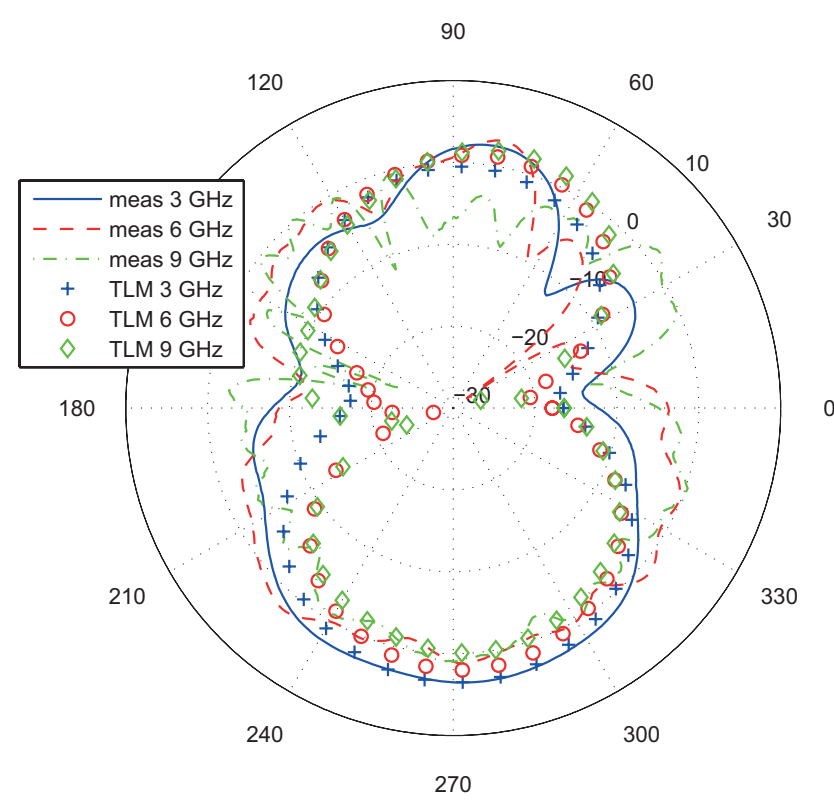

Fig. 5 Bow-tie antenna: azimuthal radiation pattern

Since measurements of antenna patterns are not given in [9], in Fig. 5 and 6 only results of our measurement and TLM calculations are shown. The differences between measurement and simulation are due to limited performance of our anechoic chamber. The influence of the test fixture is not negligible.

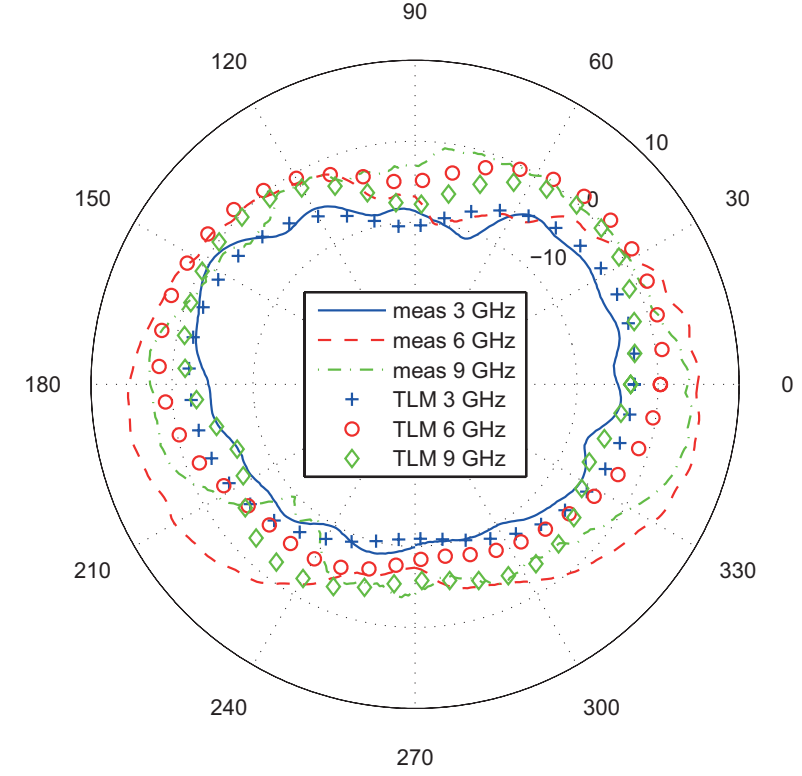

Fig. 6 Bow-tie antenna: elevation radiation pattern

\subsection{Vivaldi antenna}

This type of antenna is particularly useful for broadband transmission, because of its frequency constant omni directional radiation patter. The azimuthal and elevation patterns are shown in Fig. 7 and 8.

90

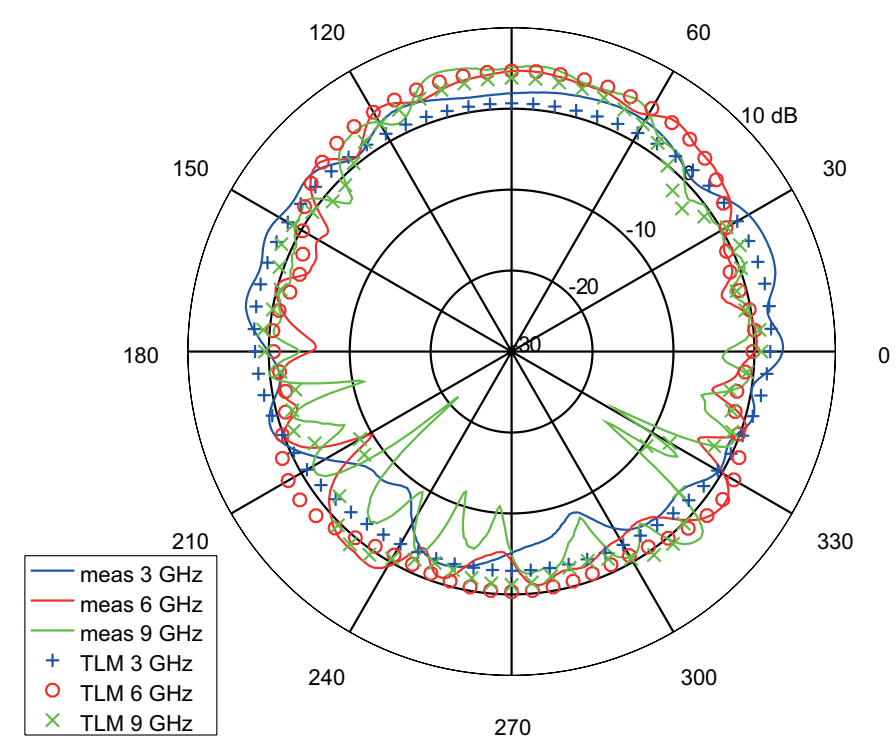

Fig. 7 Vivaldi antenna: azimuthal radiation pattern

The simulation accurately predicts the performance of the Vivaldi antenna, knowing that the differences around $270^{\circ}$ arise from our test-fixture.

From Fig. 9, it can be seen that the antipodal Vivaldi antenna is not well matched to the $50 \Omega$-SMA connector of our vector network analyzer. Hence it slightly misses the $-10 \mathrm{~dB}$ matching line. At this point the antenna shows optimization potential. Similar to the report [13], the simulation does not exactly coincide with the measurement, but follows its characteristics. 


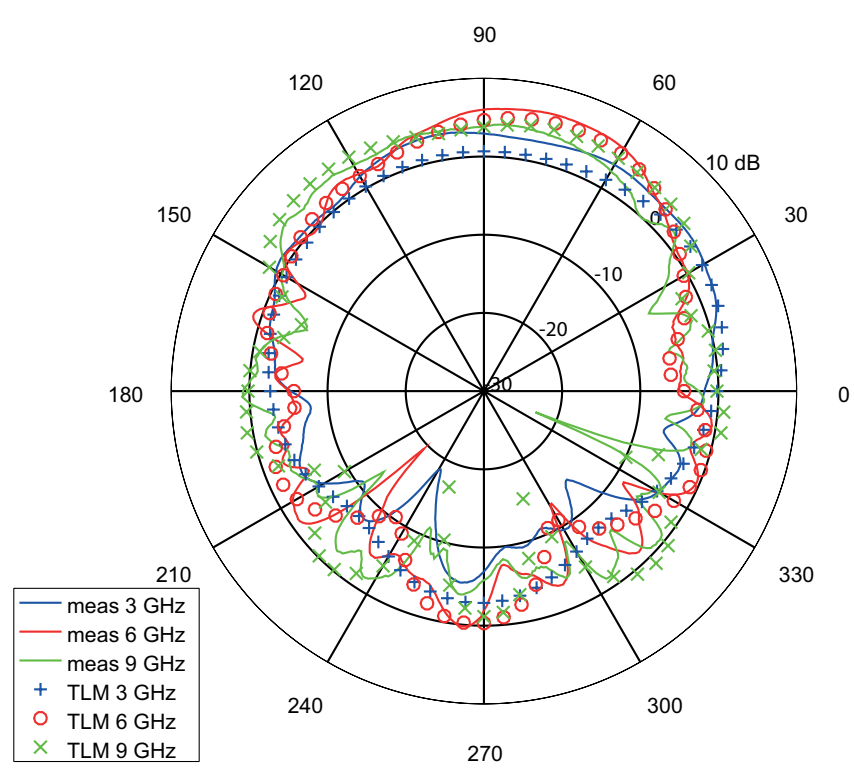

Fig. 8 Vivaldi antenna: elevation radiation pattern

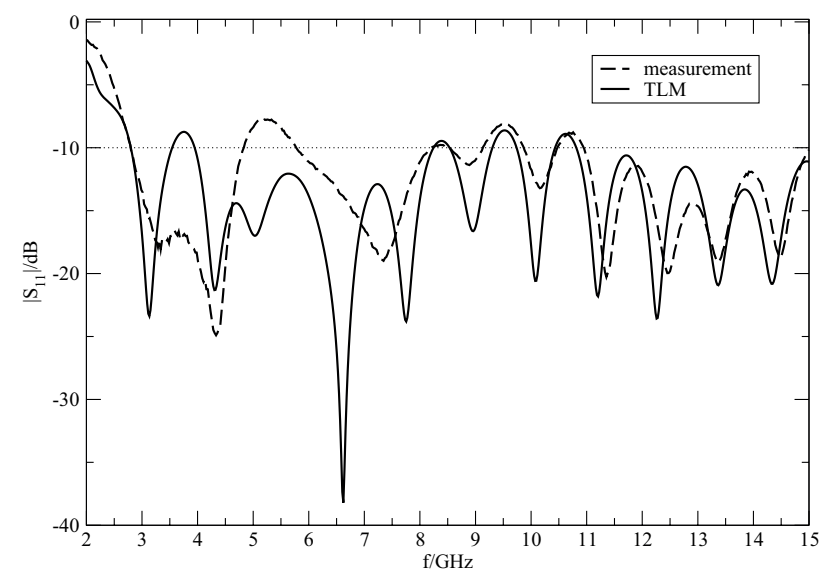

Fig. 9 Reflection coefficient $\left|S_{11}\right|$ for the Vivaldi antenna

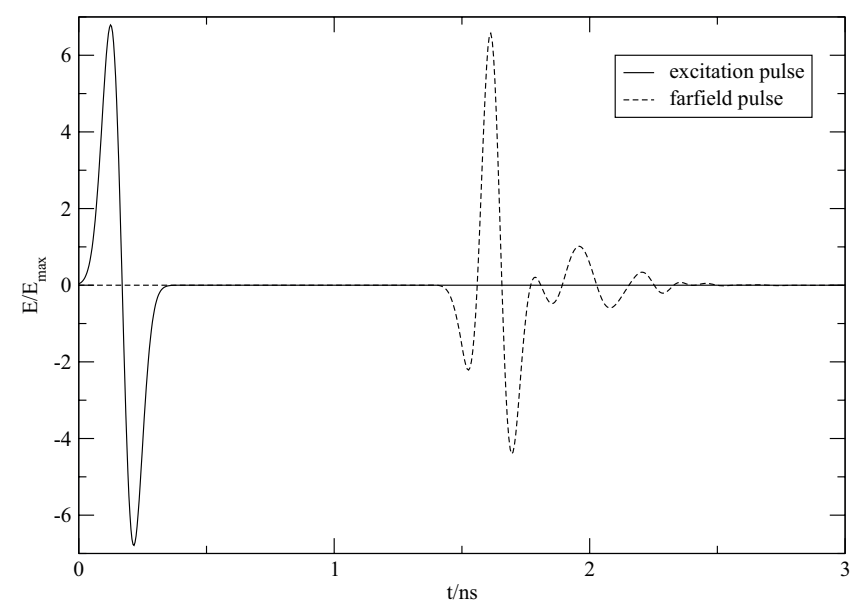

Fig. 10 Normalized radiated E-field pulse from the Vivaldi antenna

Regarding UWB performance, the pulse transmission capability of an antenna is important. Figure 10 shows a Gauss monocycle pulse as excitation signal and the transmitted pulse ( $\Theta$-polarized e-field) in the farfield $\left(\Phi=90^{\circ}, \Theta=90^{\circ}\right)$. Time and amplitude are normalized (see [7]) for better displaying. The farfield signal exhibits only small disturbances, so called ringing. This antenna is well suited for pulse transmission.

\section{Conclusions}

In this paper the capability of EPML in conjunction with the TLM method has been demonstrated by means of a double-sided printed bow-tie antenna and an antipodal Vivaldi antenna. Radiation patterns as well as scattering parameters are introduced and discussed. The feasibility of these antennas for UWB applications is shown on an example of the Vivaldi antenna by the help of its pulse transmission capability.

\section{References}

[1] Johns, P. B. "A Symmetrical Condensed Node for the TLM Method." IEEE Transaction on Microwave Theory and Techniques. 35 (4). pp. 370-377. 1987. DOI: 10.1109/TMTT.1987.1133658

[2] Collin, R. E. "Field Theory of Guided Waves." $2^{\text {nd }}$ ed., IEEE Press, Piscataway, NJ, 1991.

[3] Bérenger, J.-P. "Tree-Dimensional Perfectly Matched Layer for the Absorption of Electromagnetic Waves." Journal of Computational Physics. 127 (2). pp. 363-379. 1996. DOI: 10.1006/jcph.1996.0181

[4] Dubard, J.-L., Pompei, D. "Optimization of the PML Efficiency in 3-D TLM Method." IEEE Transactions on Microwave Theory and Techniques. 48 (7). pp. 1081-1088. 2000. DOI: 10.1109/22.848490

[5] Le Maguer, S., Ney, M. M. "Extended PML-TLM node: an efficient approach for full-wave analysis of open structures." International Journal of Numerical Modeling: Electronic Networks, Devices and Fields. 14 (2). pp. 129-144. 2001. DOI: 10.1002/jnm.402

[6] Taflove, A., Hagness, S. C. "Computational Electrodynamics: the finitedifference time-domain method." Norwood, Artech House, 2000.

[7] Luebbers, R. J., Kunz, K. S., Schneider, M., Hunsberger, F. "A FiniteDifference Time-Domain Near Zone to Far Zone Transformation." IEEE Transactions on Antennas and Propagation. 39 (4). pp. 428-433. 1991. DOI: $10.1109 / 8.81453$

[8] Gwarek, W. K., Celuch-Marcysiak, M. "A Differential Method of Reflection Coefficient Extraction from FDTD Simulations." IEEE Microwave and Guided Wave Letters. 6 (5). pp. 215-217. 1996. DOI: $10.1109 / 75.491510$

[9] Kiminami, K., Hirata, A., Shiozawa, T. "Double-Sided Printed BowTie Antenna for UWB Communications." IEEE Antennas and Wireless Propagation Letters. 3 (1). pp. 152-153. 2004. DOI: 10.1109/LAWP.2004.832126

[10] Pandey, G., Soh, P. J., Mercuri, M., Beyer, A., Vandenbosch, G. A. E., Schreurs, D. "EM-based Antenna Optimization for Health Monitoring Radar Sensor." In: "Advances in Modeling-Band Simulation" The 29th Annual Review of Progress in Applied Computational Electromagnetics, Hotel Embassy Suites, Monterey, California, USA, pp. 632-637, March 24th - March 28th, 2013.

[11] Mercuri, M., Karsmakers, P., Leroux, P., Schreurs, D., Beyer, A. "RealTime Fall Detection and Tagless Localization using Radar Techniques." In: 2015 IEEE 16th Annual IEEE Wireless and Microwave Technology Conference, (WAMICON) 2015, pp.1-3, Cocoa Beach, Florida, USA, 13-15 April 2015. DOI: 10.1109/WAMICON.2015.7120363

[12] Beyer, A., Roth, B. "Development of millimeter wave antennas in slot line technique for integrated communication systems." Antennas and Propagation Society International Symposium, 1990. AP-S. Merging Technologies for the 90's. Digest., Vol. 4, pp. 1918-1921, Dallas, Texas, USA, 7-11 May 1990. DOI: 10.1109/APS.1990.115510

[13] "The 2000 CAD Benchmark." Microwave Engineering Europe, [Online]. Available from: http://www.embedded.com/electronicsnews/4162379/The-2000-CAD-Benchmark [Accessed: 2004] 